\title{
Methylation levels of a novel genetic element, EgNB3 as a candidate biomarker associated with the embryogenic competency of oil palm
}

\begin{abstract}
The association between DNA methylation status and embryogenic competency in oil palm tissue culture was examined through Representational Difference Analysis (RDA) approach, using methylation-sensitive restriction endonucleases. "Difference Products" (DPs) of RDA derived from palms of similar genetic backgrounds but exhibiting different embryogenesis rates during the regeneration process were isolated. The DPs were sequenced using a pyrosequencing platform. To our knowledge, this is the first study profiling partial HpaII methylation sites in oil palm young leaf tissues which are potentially associated with embryogenic amenability through a genomic subtractive approach. Quantitative real-time PCR analysis demonstrated that the methylation status of a novel fragment, EgNB3, was higher in highly embryogenic leaf explants compared to low embryogenesis rate materials. These differences are likely to be contributed by the 5'-mCCGG-3' and/or 5'-mCmCGG-3' methylation patterns. Our data suggest that the differentially methylated site in EgNB3 has potential as a molecular biomarker for the screening of oil palm leaf explants for their embryogenic potentials.
\end{abstract}

Keyword: Biomarker; Embryogenic potential; Oil palm; Site-specific methylation. 